\title{
'To $B$ or not to $B$ ' Compression sutures - what a patient needs to know after the bleeding is controlled: A review
}

\author{
Kimberley GS Thornton* and R Douglas Wilson \\ Department of Obstetrics and Gynecology, University of Calgary Cumming School of Medicine, Alberta, Canada
}

\begin{abstract}
This article aims to review the maternal morbidity, as well as both short and long-term complications associated with the different types of uterine compression sutures used in managing postpartum hemorrhage. PubMed and Medline databases were non-systematically searched for identified complications of uterine compression sutures. Complications following uterine compression suture placement appear to be rare but likely are underreported. Uterine anatomical distortion and adhesions, postoperative infectious complications, fertility issues, and uterine rupture in subsequent pregnancy have been reported following uterine compression sutures. The general paucity of evidence limits the ability to make recommendations for monitoring following the use of uterine compression sutures, but possible hysteroscopy and thorough communication appear to be reasonable. A non-validated post-operative counseling checklist based on the available evidence was created to assist the hospital debriefing and counseling between physician and patient.
\end{abstract}

\section{Introduction}

Dr Christopher B-Lynch is a UK obstetrician who first reported the use of a uterine compression suture (UCS) for management of severe uterine atony in the setting of postpartum hemorrhage (PPH) [1]. Several modifications of the B-Lynch suture have been developed, two commonly reported modifications being Hayman and Cho sutures $[2,3]$. Appropriate post-operative patient counseling is required, as subsequent pregnancy risks are present, but this knowledge translation process is underutilized. The objective is to review the early and later complications related to the use of UCS for the management of PPH to incite discussion surrounding subsequent risk and aid in thorough patient counselling. A patient debriefing checklist and explanation tool has been created.

\section{Methods}

PubMed/Medline/Ovid databases were searched surrounding identified complications of UCS. Searches were initially for randomized controlled trials, but were broadened to all levels of evidence. Preference was given to published systematic reviews, with subsequent searches of the databases completed beyond the date of publication of each systematic review in an attempt to include all current literature.

\section{Results}

The three most common types of UCS are summarized for placement, advantages, and possible considerations (Table 1; Figure 1). All techniques recommend absorbable suture to minimize risk of bowel herniation through remaining loops of suture during normal postppartum involution of the uterus [1].

No Cochrane or comprehensive systematic reviews have been published regarding the incidence or management of all possible complications following UCS use. Complications are rare and potentially underreported.

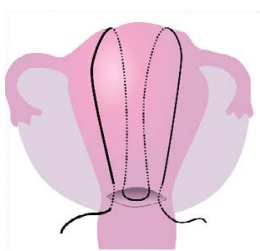

A)

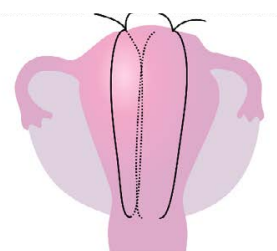

B)

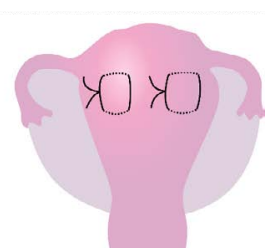

C)
Figure 1. Technique and placement of the described uterine compression suture (UCS) types.

A) B-Lynch, B) Hayman, C) Cho

\section{Postoperative complications: Anatomical distortion and infection}

Data on postoperative complications is minimal. Lower quality evidence has been reported with infectious to anatomical complications. The most common postoperative UCS morbidity reported is distortion of the uterine anatomy however there also exist reports of no observed uterine distortion. Unusual uterine shapes, vertical grooves, and uterine muscular defects with varying sizes and depths of defect have been reported [4-8]. Poor obstetrical outcomes have been reported related to observed uterine thinning and scarring at UCS sites, with either absent or significantly decreased endometrium or myometrium thickness [68]. Uterine necrosis has been reported along the lines of UCS placement

${ }^{\star}$ Correspondence to: Kimberley GS Thornton, Department of Obstetrics and Gynecology, University of Calgary Foothills Medical Centre: Room 428, North Tower, 1403 29th Street NW, Calgary, AB, Canada T2N 2T9, E-mail: kimberley.thornton@albertahealthservices.ca

Key words: maternal morbidity, postpartum hemorrhage, secondary infertility, uterine compression sutures, uterine rupture, uterine synechiae

Received: November 05, 2018; Accepted: November 16, 2018; Published: November 19, 2018 
Table 1. Summary of advantages and considerations for different types of uterine compression sutures (UCS) used in the management of post-partum hemorrhage [1-3]

\begin{tabular}{|c|l|l|}
\hline Suture Type & \multicolumn{1}{|c|}{ Advantages } & \\
\hline B-Lynch & $\begin{array}{l}\text { - one continuous suture creates even pressure } \\
\text { - can use Bakri concurrently } \\
\text { - only type with procedure registry } \\
\text { - hysterotomy allows exploration of uterine cavity }\end{array}$ & - requires hysterotomy \\
\hline Hayman & - does not require hysterotomy & - fast to place \\
\hline Cho & - can allow more targeted compression to specific areas of atony & $\begin{array}{l}\text { - risk of unidentified obstruction of cervical os } \\
\text { - cannot use Bakri concurrently }\end{array}$ \\
\hline & - does not require hysterotomy & $\begin{array}{l}\text { - cannot use Bakri concurrently } \\
\text { - possible risk of infection if interrupts uniform involution } \\
\text { - multiple needle punctures can result in increased bleeding }\end{array}$ \\
\hline
\end{tabular}

in the early postoperative period, associated with a risk of postpartum hysterectomy $[4,6]$.

Pyometra and hematometra have been reported [4]. An increased rate of postpartum endometritis following UCS placement was significant when compared to no UCS for emergency cesarean sections by Suzuki et al. [5]. Variables such as total time of ruptured membranes, number of exams, and surgical preparation were not controlled for in the analysis [5].

\section{Future fertility}

There is limited research about investigations or treatment of women following UCS who desired subsequent pregnancy but have secondary infertility. Erosion of B-Lynch sutures through the uterine wall has been observed, both in the early postpartum period and later with secondary infertility $[4,9]$. Following the removal of suture material via operative hysteroscopy, a successful pregnancy has been reported [9].

A systematic review assessed menstrual or fertility outcomes for women having had UCS for PPH [10]. A total of 125 women with variable outomes reported $91.5 \%$ had normal resumption of menses within six months following UCS, and $85.71 \%$ of women who had expressed a desire for subsequent pregnancy were successful [10]. A case control study found no statistically significant difference in miscarriage, tubal pregnancy rate, or preterm delivery in women who had received UCS at cesarean section compared to age and parity matched controls with cesarean section without UCS [11]. Additional UCS literature has reported a range from $38.1 \%$ to $47.4 \%$ live birth rates in women expressing desire for subsequent pregnancy $[12,13]$.

Hysteroscopy, hysterosalpingogram, sonohysterogram, or a combination identified the most common finding post UCS was uterine synechiae, but normal cavities were also observed [4]. Uterine cavity investigations were completed from fifteen days to two years postpartum [4]. A separate retrospective study reported a normal uterine cavity in $68 \%$ of women with a $16 \%$ rate of uterine synechiae on hysteroscopy within 3-6 months postpartum [14]. Operative hysteroscopic removal of the synechiae within 3-6 months post UCS reported sucessful spontaneous pregnancy outcomes [14].

\section{Uterine rupture}

The reports of uterine rupture following UCS are rare, with uterine rupture at 20,32, and 33 week gestations [6-8]. These cases had intraoperative findings of vertical, anterior uterine rupture with the rupture edges documented as appearing thinned and/or scarred. There was no evidence of lower uterine segment compromise seen. In all three case reports, it was proposed that the fundal defect was associated with the location of the previous UCS [6-8].

\section{Intraabdominal adhesions}

No systematic review for the rate of intraperitoneal adhesions following UCS was identified. A seven year cohort for the B-lynch suture was reported in 28 cases with cesaeran delivery [15]. From this cohort, seven women had a repeat cesarean delivery and three were found to have abdominal adhesions although minimal further qualification of severity was provided [15]. A statistically significant increased rate of abdominal adhesions (at the time of repeat section) with previous UCS as compared to age and parity matched controls has been reported [11]. No significant difference in operative time or estimated blood loss was associated with these increased adhesions [11].

\section{Discussion}

Overall the data surrounding complications of UCS tends to be low quality (case reports or retrospective studies), with significant heterogeneity and rare outcomes. This paucity further justifies a need for more research and discussion amongst obstetricians surrounding future clinical management guidelines for patients after UCS for PPH.

Uterine anatomical distortions and their associated risk are based on limited data, creating a selection bias. The clinical implications of these anatomical findings have not been determined. Cases reporting uterine necrosis have used either multiple UCS or simultaneous multiple UCS types [4]. Therefore, if additional sutures of multiple techniques are required then the risk for increased suture tension, ischemia, and uterine necrosis is possible $[4,6]$. Monitoring for postpartum infection is recommended. There does not appear to be an increased risk for postpartum endometritis, and antibiotic prophylaxis is not presently justified [5].

Uterine cavity synechiae prevalence following UCS has not been delineated, but is commonly reported $[4,9,14]$. The post llUCS prevalence rate of synechiae is limited by several factors as studies were observational or case reports with only a fraction of UCS women having further evalaution. The clinical significance of synechiae has not been well investigated, but treatment of identified synechiae following UCS has resulted in positive pregnancy outcomes [9,14]. Reports of successful pregnancy following synechiae resoution with operative hysteroscopy highlights the consideration for screening women with hysteroscopy or uterine cavity imaging post UCS $[9,14]$. Some evidence suggests timing this at 3-6 months postpartum [14]. Using WHO criteria for justifying screening tests, the test is relatively low risk, the identification of synechiae is clear, and an accepted treatment exists [16]. The cost and risk benefit policy requires further study, especially as the majority of cases investigated with hysteroscopy did not have synechiae [14]. Secondary infertility in women who have received UCS likely should include assessment of the uterine cavity due to the possible risk for intrauterine synechiae. 
Post UCS fertility outcomes for women are reported. However, data is limited by small numbers and large variation. Outcomes are focused on patients that desired a subsequent pregnancy, with large follow-up gaps for patients who desired pregnancy at a much later date [10]. Only one study sought to review subsequent pregnancy complications (miscarriage or preterm delivery) leaving another knowledge gap [11].

The risk of uterine rupture following UCS is undetermined. The total number of UCS procedures performed and subsequent uterine rupture are likely underreported. Multiple authors have suggested a relationship between impaired uterine healing and later uterine rupture [6-8]. Uterine rupture has been reported with a history of prolonged postoperative pyrexia following the original cesarean and UCS, but no further explanation or details were provided [7]. This asks the question whether tissue ischemia or injury following UCS placement, with postpartum infection or excessive tension on the sutures, could be associated with uterine weakness and possible rupture.
Routine recommendation for repeat cesaeran section in subsequent pregnancies after UCS would not be evidence based given the absence of a calculable risk of rupture. However, it is reasonable and appropriate to discuss with the patient that uterine ruptures have been reported in subsequent pregnancies when dicussing the options of either repeat cesarean delivery or a trial of labour for vaginal birth.

The increased risk of intraperitoneal adhesions associated with previous UCS use has minimal clinical implications with no statistically different blood loss or increased operative time compared with repeat cesarean delivery [11]. Meticulous surgical technique to avoid adhesions is still standard of care.

Maternal morbidity risks following UCS placement for $\mathrm{PPH}$ management should require a hospital-based debriefing between obstetrician and patient before discharge. Figure 2 outlines an example of a non-validated patient counseling checklist based on the reported operative and post-operative morbidity.

Patient Name:

Date:

$\checkmark$ What happened at your delivery? Why was a uterine compression suture needed?

$\checkmark$ Describe the uterine compression suture used:

SHORT TERM CONSIDERATION

$\checkmark$ There is a small risk for infection or breakdown at the site of the sutures that will be observed for carefully

$\checkmark$ Normal recovery factors will continue to be monitored in your postpartum period

LONG TERM CONSIDERATIONS To Remember
$\nabla$ The rate and outcomes of future fertility are very favorable based on a small number of
available studies
$\nabla$ The uterus may heal in an abnormal fashion with thinning or scarring underneath the
compression sutures
$\diamond$ This may put you at risk for the uterus breaking down at the site of the suture in a
future pregnancy, but this emergency has only happened in a very small number of
case reports
$\diamond$ Having multiple compression suture types or an infection in your recovery period may
increase this risk
$\nabla$ The cavity of the uterus has a small chance of forming scar tissue within itself as it heals
after compression suture
$\diamond$ This may cause challenges with fertility in the future, but has only been seen in a small
number of case reports
$\diamond$ If there is urgency to a future pregnancy, a test to look inside the uterus (called a
hysteroscopy) or special imaging of your uterine cavity (called a hysterosalpingogram)
may be reasonable at 3-6 months postpartum, but this should be discussed with your
obstetrician/gynecologist
$\nabla$ Method of delivery in a future pregnancy should be discussed with your obstetrical care
provider
$\diamond$ There is no evidence that suggests a repeat cesarean is safer than a trial of labor with
a history of compression sutures alone
$\diamond$ Specific factors (like other intraoperative findings, your healing postpartum, or your
obstetrical history) may lead to recommendation of repeat cesarean section for future
delivery
Have we answered all your questions?

Figure 2. outlines an example of a non-validated patient counselling checklist based on the reported operative and post-operative morbidity 


\section{Conclusion}

Uterine preservation following UCS use for PPH requires hospitalbased patient debriefing and counseling regarding the risks of postoperative complications, possible fertility issues, and uterine rupture risk. More evidence is required to better guide future clinical practice in postpartum and subsequent pregnancy following UCS placement for $\mathrm{PPH}$.

\section{References}

1. Allam MS, B-Lynch C (2005) The B-Lynch and other uterine compression suture techniques. Int J Gynaecol Obstet 89: 236-241. [Crossref]

2. Cho JH, Jun HS, Lee CN (2000) Hemostatic suturing technique for uterine bleeding during cesarean delivery. Obstet Gynecol 96: 129-131. [Crossref]

3. Hayman RG, Arulkuraman S, Steer PJ (2002) Uterine compression sutures: surgical management of postpartum hemorrhage. Obstet Gynecol 99: 502-506. [Crossref]

4. Amorim-Costa C, Mota R, Rebelo C, Silva PT (2011) Uterine compression sutures for postpartum hemorrhage: is routine postoperative cavity evaluation needed? Acta Obstet Gynecol Scand 90: 701-706. [Crossref]

5. Suzuki Y, Matsuzaki S, Mimura K, Kumasawa K, Tomimatsu T, et al. (2017) Investigation of perioperative complications associated with use of uterine compression sutures. Int J Gynaecol Obstet 139: 28-33. [Crossref]

6. Harlow FH, Smith RP, Nortje J, Anigbogu BO, Tyler X (2017) Catastrophic uterine rupture associated with placenta accreta after previous B-Lynch sutures. J Obstet Gynaecol. Ahead of print.
7. Petchor K, Richards B, Paterson H (2010) Antenatal catastrophic rupture at 32 weeks gestation after previous B-lynch suture. BJOG 117: 889-891. [Crossref]

8. Shilpa D, Bhaskar M, Arihant M (2014) Post B-lynch uterine rupture: case report and review of the literature. J Obstet Gynaecol India 64: 362-363. [Crossref]

9. Staff S, Uotila J, Tinkanen H (2014) Secondary infertility after B-Lynch suture indication for hysteroscopy as a first-line diagnostic procedure? Acta Obstet Gynecol Scand 93: 721-723. [Crossref]

10. Doumouchtsis SK, Nikolopouls K, Talaulikar VS, Krishna A, Arulkumaran S (2014) Menstrual and fertility outcomes following the surgical management of postpartum hemorrhage: a systematic review. BJOG 121: 382-388. [Crossref]

11. An GH, Ryu HM, Kim MY, Han JY, Chung JH, et al. (2013) Outcomes of subsequent pregnancies after uterine compression sutures for postpartum hemorrhage. Obstet Gynecol 122: 565-570. [Crossref]

12. Liu S, Mathur M, Tagore S (2014) Complications and pregnancy outcome following uterine compression suture for postpartum hemorrhage: a single centre experience. $J$ Obstet Gynaecol 34: 383-386. [Crossref]

13. Tadakawa M, Sugawara J, Saito M, Nishigori H, Utsunomiya H, et al. (2014) Fertility and pregnancy outcomes following B-Lynch sutures for post-partum hemorrhage. $J$ Obstet Gynaecol Res 41: 559-564. [Crossref]

14. Jamard A, Turck M, Cheret-Benoist A, Dreyfus M, Benoist G (2014) Risk of uterine synechiae following uterine compression sutures during postpartum hemorrhage. Gynecol Obstet Fertil 42: 681-685. [Crossref]

15. Baskett TF (2007) Uterine compression sutures for postpartum hemorrhage: efficacy, morbidity, and subsequent pregnancy. Obstet Gynecol 110: 68-71. [Crossref]

16. Wilson JMG, Jungner G (1968) Principles and practices of screening for disease. World Health Organization. Public Health Papers No. 34

Copyright: $\odot 2018$ Thornton KGS. This is an open-access article distributed under the terms of the Creative Commons Attribution License, which permits unrestricted use, distribution, and reproduction in any medium, provided the original author and source are credited. 